\title{
Blood Flow Measurements With Magnetic Resonance Phase Velocity Mapping
}

George P. Chatzimavroudis

Cleveland State University

Follow this and additional works at: https://engagedscholarship.csuohio.edu/encbe_facpub

Part of the Biomedical Devices and Instrumentation Commons, and the Transport Phenomena

\section{Commons}

How does access to this work benefit you? Let us know!

\section{Publisher's Statement}

NOTICE: this is the author's version of a work that was accepted for publication in

Measurement. Changes resulting from the publishing process, such as peer review, editing, corrections, structural formatting, and other quality control mechanisms may not be reflected in this document. Changes may have been made to this work since it was submitted for publication. A definitive version was subsequently published in Measurement, [37, 3, (April 2005)] DOI 10.1016/j.measurement.2004.10.001

\section{Original Citation}

Chatzimavroudis, G. P. (2005). Blood flow measurements with magnetic resonance phase velocity mapping. Measurement, 37(3), 201-212. doi:10.1016/j.measurement.2004.10.001

\section{Repository Citation}

Chatzimavroudis, George P., "Blood Flow Measurements With Magnetic Resonance Phase Velocity Mapping" (2005). Chemical \& Biomedical Engineering Faculty Publications. 75.

https://engagedscholarship.csuohio.edu/encbe_facpub/75

This Article is brought to you for free and open access by the Chemical \& Biomedical Engineering Department at EngagedScholarship@CSU. It has been accepted for inclusion in Chemical \& Biomedical Engineering Faculty Publications by an authorized administrator of EngagedScholarship@CSU. For more information, please contact library.es@csuohio.edu. 


\title{
Blood flow measurements with magnetic resonance phase velocity mapping
}

\author{
George P. Chatzimavroudis * \\ Department of Chemical and Biomedical Engineering, Cleveland State University, and Section of Cardiovascular \\ Imaging, Division of Radiology, The Cleveland Clinic Foundation, 2121 Euclid Avenue, SH455, Cleveland, OH 44115, USA
}

\section{Introduction}

Hydrogen-based magnetic resonance (MR) imaging provides structural, functional, and flow information non-invasively. The technique is based on the interaction of the magnetic moment

\footnotetext{
* Tel.: +1 216687 5396; fax: +1 2166879220 .

E-mail address: g.chatzimavroudis@csuohio.edu
}

of hydrogen nuclei (protons) with applied magnetic fields. If a mass of protons is placed inside a static magnetic field, the magnetic moments of the protons tend to align parallel and anti-parallel with the direction of the static field. The protons inside the magnetic field are in a complex rotational motion, called precession, with a frequency that depends on the strength of the magnetic field. Using radio-frequency pulses with a frequency 
equal to this precession frequency, the protons can be energetically excited. While they return to equilibrium, they emit a signal that is used to reconstruct an image. By applying a combination of magnetic field gradients in the $x$-, $y$-, and $z$-direction (by convention the frequency encoding direction, the phase encoding direction, and the slice selection direction, respectively (Fig. 1)), the position of the excited protons can be spatially encoded. The raw data is in the frequency domain ( $k$-space). Every time the protons are excited and then return to equilibrium, the received signal fills one or more lines of $k$-space. After the $k$-space is filled, an inverse two-dimensional Fourier transform reconstructs the image in the space domain.

An important feature of MR is its ability to measure flow velocity in any spatial direction using a technique called MR phase velocity mapping (PVM). By applying the proper bipolar magnetic field gradients (Fig. 1), the velocity of the protons can be encoded in the phase of the received signal [1]. This phase-velocity encoding follows a linear equation:

$\phi=\left(\gamma M_{1}\right) v=\left(\gamma \int_{0}^{\mathrm{TE}} G(t) t \mathrm{~d} t\right) v=\left(\gamma A_{\mathrm{g}} T\right) v$

where $\phi$ is the phase of the received signal, $\gamma$ is the gyromagnetic ratio $[\mathrm{Hz} / \mathrm{T}], v$ is the velocity (as-

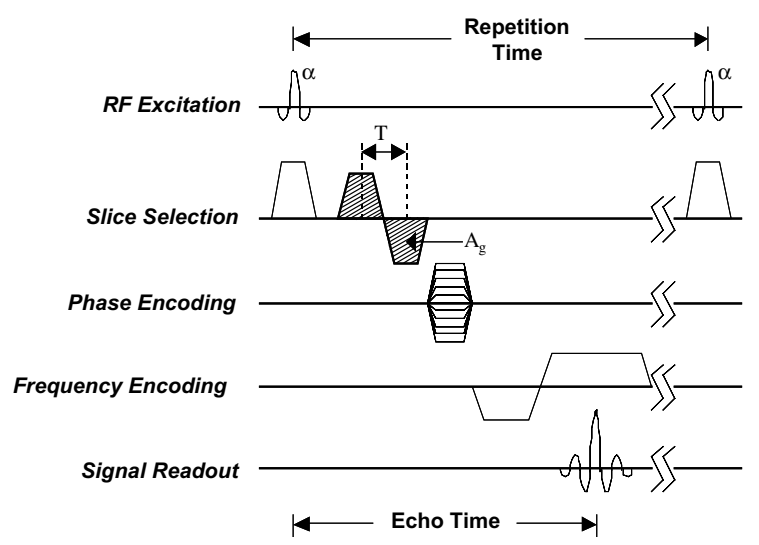

Fig. 1. A simplistic diagram of an unsegmented gradient-echo MR sequence with a bipolar gradient (shaded area) for velocity encoding in the slice selection direction ( $\alpha$ : flip angle, $A_{\mathrm{g}}$ : bipolar gradient single lobe area, $T$ : time between the lobe centers in the bipolar gradient). sumed constant) $[\mathrm{m} / \mathrm{s}], M_{1}$ is the first moment of the gradient waveform $\left[\mathrm{T} \mathrm{s}^{2} / \mathrm{m}\right]$ at the echo time (TE), $G(t)$ is the magnetic field gradient $[\mathrm{T} / \mathrm{m}]$, $A_{\mathrm{g}}$ is the "area" of each lobe of the bipolar gradient $[(\mathrm{T} / \mathrm{m}) \mathrm{s}]$, and $T$ is the time between the centers of the two lobes of the gradient [s]. The magnitude of the acquired signal is used to reconstruct the magnitude (structural) image, whereas the phase of the signal can be used to reconstruct the phase (velocity) map.

The ability of MR to characterize and quantify flow has found wide application in the clinical field for blood flow measurements in the aorta, pulmonary artery, venae cavae, ventricles, and various arteries and veins [2-9]. MR has also been of value in non-biomedical applications, such as to characterize the velocity profiles of pure fluids and suspensions [10], to study flow in porous media [11], to visualize flow in fixed-bed reactors [12], and as a rheological technique [13] and viscometer [14]. Studies have shown that conventional unsegmented $k$-space MR PVM is accurate and precise, with errors of less than $10 \%$ under both steady and pulsatile flow conditions [15-20]. Clinical studies evaluating the technique for blood flow characterization or quantification found good correlations with Doppler ultrasound and flowmetric techniques $[6,16,21]$.

This review will focus on the application of MR PVM to two clinical problems: the quantitative diagnosis of heart valve regurgitation and the evaluation of the energetic performance of the total cavo-pulmonary connection. Recent studies on the potential of MR PVM for rapid velocity acquisitions will also be discussed.

\section{Heart valve regurgitation}

The human heart has four one-way valves that regulate blood circulation. Two of these valves, the aortic and the mitral, are located in the left side of the heart (Fig. 2). The aortic valve is located between the left ventricle and the aorta, and allows blood to flow during systole from the heart to all arteries that supply blood to organs and muscles. Under normal conditions, the aortic valve is completely closed during diastole. The mitral valve is 


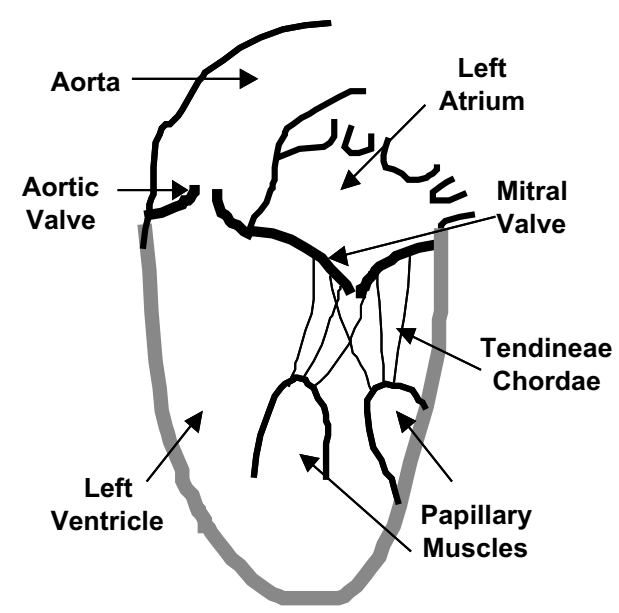

Fig. 2. A schematic of the left side of the heart.

located between the left atrium and the left ventricle, and allows blood to fill the left ventricle during diastole. Under normal conditions, the mitral valve is completely closed in systole. However, because of a variety of reasons, these valves may not close completely allowing some blood to flow in the opposite direction. This disease is called valvular regurgitation and has a direct effect on cardiac function. In severe cases, it leads to death if it remains untreated.

Knowledge of the severity of regurgitation is diagnostically very important for proper patient management and for optimizing the time for valve replacement surgery. In the past, a number of clinical approaches have been used to diagnose the severity of regurgitation. Unfortunately, these approaches usually lack reliability. Angiography [22] has been traditionally used to diagnose the disease, but it is invasive, only semi-quantitative, and involves radiation exposure. A variety of echocardiographic and MR techniques [23-29] have also been evaluated to assess regurgitation focusing on the jet side of the regurgitant valve. Unfortunately, these techniques are accompanied by technical limitations. One of the main problems is that they do not provide information about the main variable of interest, which is the volume of blood that regurgitates through the valve.

The regurgitant volume has been identified as an important index of the severity of regurgitation.
The first approach to determine the regurgitant volume was through volumetric MR imaging techniques $[21,30]$, from the difference between the left and right ventricular stroke volumes. Although the combination of left and right ventricular volumes with systolic and diastolic aortic and pulmonic flows can provide an estimate for the regurgitant volumes of the heart valves, this approach is not reliable in the presence of multi-valvular disease. Furthermore, the volumetric techniques estimate the regurgitant volume by combining measurements at different sites in the heart and not focusing on the site of interest, which is the regurgitant valve.

One of the first promising approaches to directly measure the mitral regurgitant volume was the proximal isovelocity surface area (PISA) method [31]. Although PISA can provide an estimate of the regurgitant volume, the reliability and accuracy of the method can be severely affected by geometric uncertainties and by the complex nature of the flow field in the left ventricle due to the interaction of the regurgitant flow field with the aortic outflow field [32].

\section{Quantification of the aortic regurgitant volume with MR PVM}

MR PVM can provide accurate flow quantification in a tube from through-plane fluid velocity measurements. Since the ascending aorta is a tube, it is possible to quantify aortic blood flow by placing an imaging slice perpendicular to the long-axis of the aorta and measuring the through-plane velocity (Fig. 3). The instantaneous aortic flow rate can be calculated by integrating this velocity over the aortic cross-sectional area. Then, an integration of this flow rate over diastole can finally provide the aortic regurgitant volume.

Initial in vivo investigations [33] on the clinical potential of this single slice MR PVM method showed the presence of high negative flow rates during diastole in the ascending aorta, indicating possible aortic regurgitation. Subsequent clinical studies [34] showed a fair correlation between the MR PVM regurgitant volume results and qualitative classification using aortography. With a 


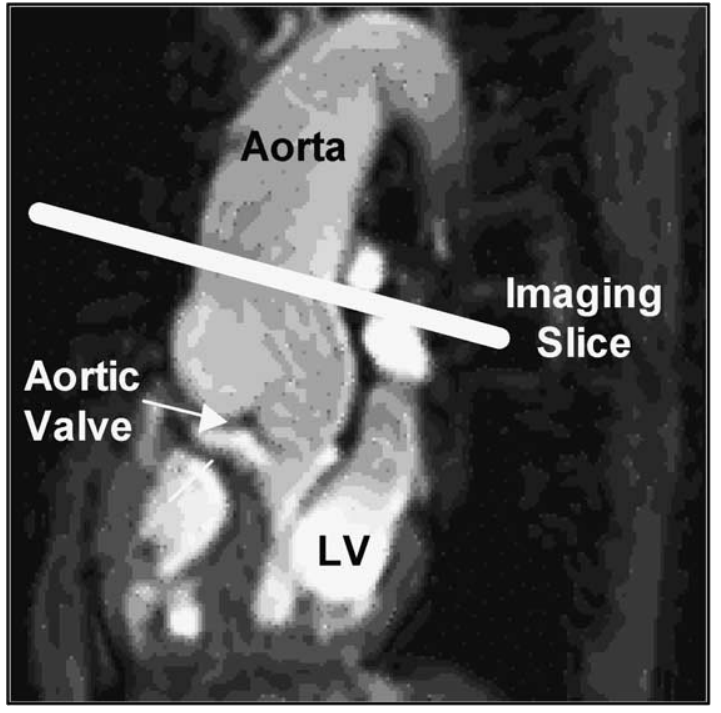

Fig. 3. The principle of the single slice method to quantify the aortic regurgitant flow. The reverse diastolic flow through the slice will primarily flow through the regurgitant aortic valve (LV: left ventricle).

$10 \mathrm{~mm}$ thick transverse slice placed in the ascending aorta at a level $1 \mathrm{~cm}$ below the innominate artery, the MR PVM results of the regurgitant volume showed good correlation with those using the ventricular volumetric MR method $(r=0.97)$ [21]. The reproducibility of the MR PVM data was also good $(r>0.97)$ [21]. The rather qualitative evaluation of the single slice technique continued using thinner slices $(5 \mathrm{~mm})$ in the ascending aorta showing small interobserver and intraobserver variations in measuring the regurgitant fraction (the ratio of the aortic regurgitant volume to the left ventricular stroke volume). The MR PVM results showed good correlation with those from volumetric MR measurements and parallel trends with echocardiographic or aortographic grading of aortic regurgitation [35]. Similarly good correlations between MR PVM results and volumetric MR results ( $r=0.97$ for the stroke volume) were found when the imaging slice was moved to the aortic valve level [36]. These studies showed that MR PVM has high potential in quantifying the aortic regurgitant volume. However, because of the variety of slice location and imaging parameters, the effects of a number of functional, fluid mechanical, and technical factors on the reliability of the technique remained unknown. Factors such as the aortic compliance, the coronary artery flow and the flow environment in the vicinity of the regurgitant orifice could have serious effects on the accuracy of the regurgitant flow measurements. These effects were studied both in vitro and in vivo.

In vitro [17], measurements were performed with a slice perpendicular to the long axis of a compliant model of the aortic root (Fig. 4), including a regurgitant aortic valve and the coronary arteries. The most accurate measurements (closest agreement between the measured and true flow values) were taken when the slice was placed between the aortic valve annulus and the coronary ostia $\left(r^{2}=0.995\right)$ (Fig. 5). When the slice was placed beyond the coronary ostia, coronary flow and aortic compliance (Fig. 6) negatively affected the accuracy of the measurements. The errors in the measurements (with respect to the known true flow values) were found to closely follow the magnitude of coronary flow and aortic compliance.

Subsequently, the single slice technique was implemented in four healthy volunteers and 19 patients with aortic regurgitation, varying from mild to severe [8]. In 13 of the patients, the slice was placed at three locations: between the aortic valve

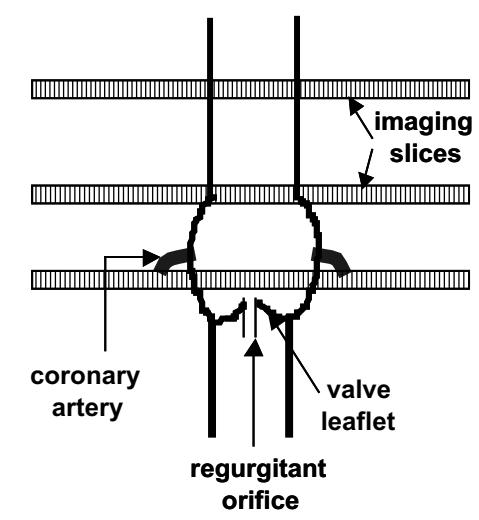

Fig. 4. A schematic of the compliant in vitro model of the aortic root. The imaging slice was positioned at different locations to investigate for the effect of coronary artery flow, aortic compliance, and the flow environment in the vicinity of the valve on the single slice measurement of the regurgitant flow. 


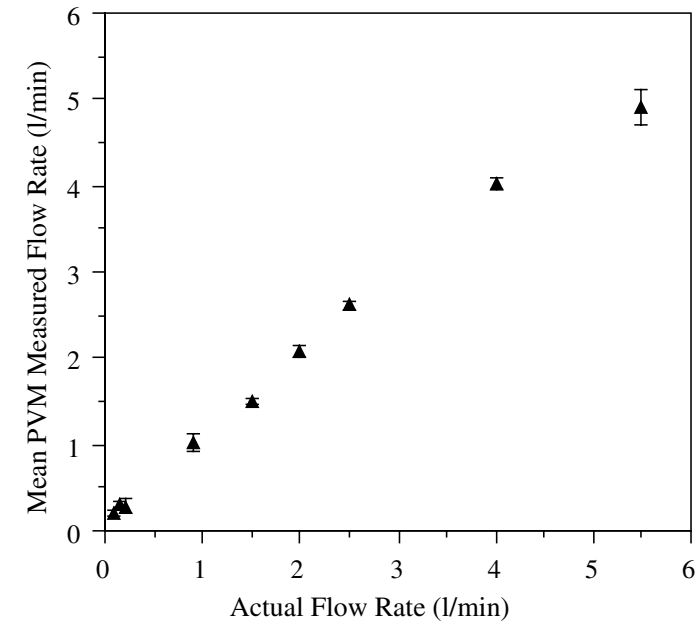

Fig. 5. In vitro MR PVM steady regurgitant flow measurements with a slice placed $0.5 \mathrm{~cm}$ from the regurgitant orifice (between the valve and the coronary ostia).

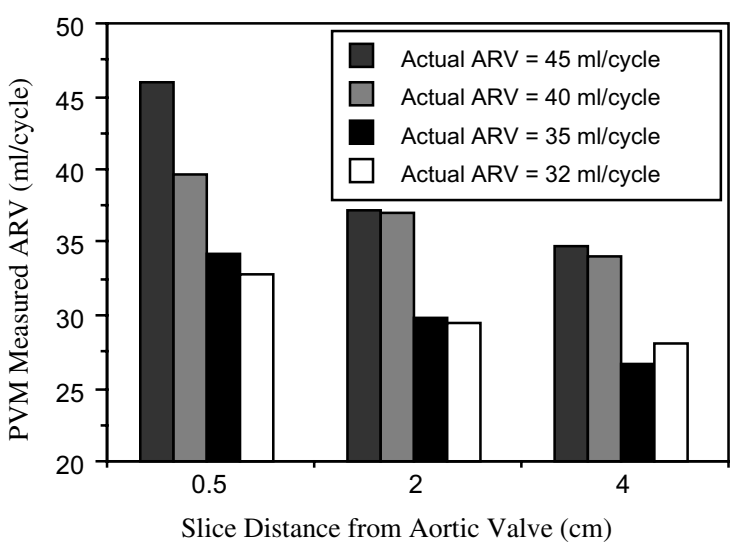

Fig. 6. MR PVM measured aortic regurgitant volume (ARV) as a function of slice location, for actual ARVs of 32, 35, 40 and $45 \mathrm{ml} /$ cycle. The measured ARV decreases as the distance between the imaging slice and the valve increases because of the effect of aortic compliance.

and the coronary ostia; at the sinotubular junction; and $2 \mathrm{~cm}$ above the sinotubular junction. In the remaining six patients, only one measurement was taken as close as technically possible to the aortic valve. The MR PVM measurements of the regurgitant volume were compared with angiographic and echocardiographic grading of aortic regurgitation. Fig. 7 shows the measured aortic

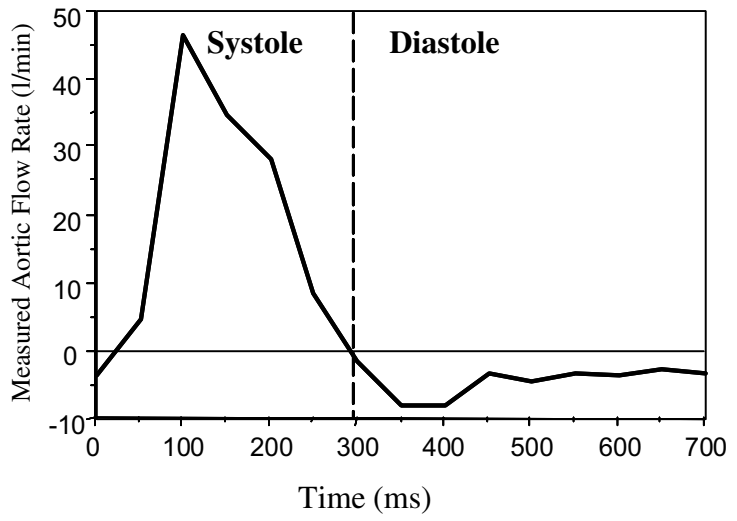

Fig. 7. Measured aortic flow waveform with an imaging slice placed in the ascending aorta of a patient of aortic regurgitation. There is considerable negative (reverse) flow activity during diastole, indicative of flow through the regurgitant valve.

flow waveform in the case of a patient with regurgitation. The measured regurgitant volume in all healthy subjects was zero. The MR PVM results correlated well with the angiographic and echocardiographic grading data. The repeatability of the MR PVM results was excellent and interobserver variability very small $\left(r^{2}=0.987, p\right.$-value $\left.=0.455\right)$. The measured regurgitant volume decreased as the distance of the slice from the aortic valve increased, due to aortic compliance, in agreement to the previous in vitro results. Even for severe severity cases of regurgitation, the difference in the measured regurgitant volume between the closest and the most distant location from the aortic valve was as high as $30 \%$. Close to the valve, blood flow acceleration did not affect the accuracy of the velocity measurements. Because of the difficulty in determining the in vivo accuracy of the regurgitant volume measurements (due to the absence of any other technique to provide such data), the reliability of the single slice technique was investigated by determining its precision [20]. In three healthy volunteers and seven patients with aortic regurgitation, single slice measurements in the ascending aorta were acquired twice. Comparison between the flow volume results from these two measurements showed high levels of precision $\left(r^{2}=\right.$ 0.993), confirmed statistically. The mean difference in the measured flow volumes between the two series of measurements was $<1 \mathrm{ml} /$ beat. 
The above results suggest that the most accurate measurements of the regurgitant volume should be expected with a slice as close to the valve as technically possible. However, one of the problems of this strategy is the fact that the valve annulus moves during the cardiac cycle. This motion may affect the reliability of proper slice placement and may introduce errors in the velocity measurements. Nevertheless, newer corrective techniques have the potential to reduce or even eliminate these problems. One of these techniques involves an adaptation of slice position according to heart motion [37]. The basal cardiac plane is first marked and the slice position (offset and angulation) is corrected based on the position of this basal plane throughout the cardiac cycle. The measured through-plane velocities are corrected to account for this basal motion. A preliminary clinical study implementing this corrective technique in 12 patients with aortic regurgitation [38] showed that the aortic annulus moved approximately $8 \mathrm{~mm}$ in the through-slice direction. The difference in the measured regurgitant fraction between implementing and not implementing this corrective technique was approximately $60 \%$ for mild regurgitation, $15 \%$ for moderate regurgitation, and $7 \%$ for severe regurgitation.

The ability of MR PVM to quantify the flow rate in a vessel by measuring the through-slice velocity component has provided a unique method to quantify the aortic regurgitant volume. Knowledge on proper slice positioning combined with the development of techniques to correct for cardiac motion are contributing to establishing this approach as the diagnostic method of choice.

\section{Quantification of the mitral regurgitant volume with MR PVM}

The quantification of the mitral regurgitant volume is more complicated than that of the aortic regurgitant volume. Although a single imaging slice placed in the ascending aorta is adequate to measure the regurgitant flow in aortic regurgitation, the same is not true in mitral regurgitation, because of the interaction between the aortic outflow and the regurgitant flow in the vicinity of the mitral valve. In an indirect way, Fujita et al. [39] quantified the mitral regurgitant volume from the difference between the mitral diastolic inflow volume (measured with a single slice placed in the mitral valve) and the aortic systolic flow volume (also measured with a single slice in the aortic root). The regurgitant fraction correlated well with echocardiographic grading of mitral regurgitation $(r=0.87)$. The limitation of this approach is that it is not valid if the patient has both mitral and aortic regurgitation.

Since measuring the regurgitant flow with a single slice is more complicated and less reliable in the case of mitral regurgitation compared to the case of aortic regurgitation, a more sophisticated method is required. As mentioned previously, MR PVM provides measurements of all three spatial components of the velocity vector in an imaging slice. Then, by acquiring a number of contiguous imaging slices with all three components of the velocity measured, a control volume in a form of an imaginary box surrounding the orifice can be considered (Fig. 8). The velocity through the faces

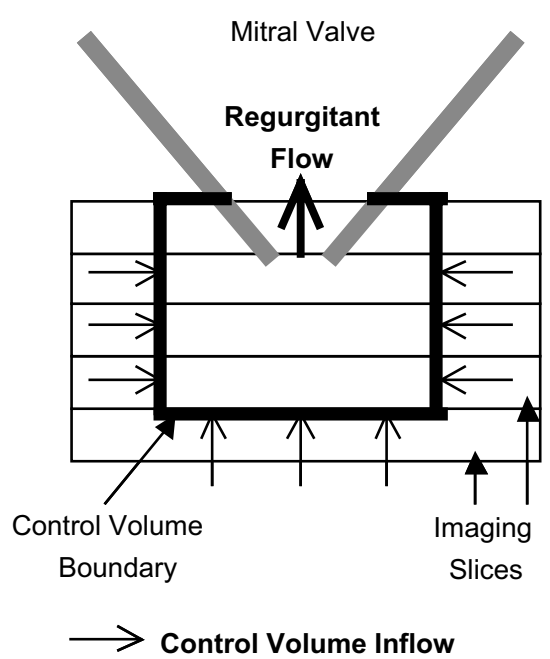

Fig. 8. The multi-slice control volume method. A number of contiguous imaging slices are positioned in the mitral valve region and all three spatial velocity components are measured. Then, because of the principle of mass conservation, the net inflow through the drawn boundaries of the control volume (shown here as a two-dimensional drawing; in reality it is a three-dimensional imaginary box) will be equal to the regurgitant flow through the valve orifice. 
of this control volume can be integrated over the total inflow area to calculate the flow rate. Based on the principle of mass conservation the net inflow into the control volume will be equal to the flow through the regurgitant orifice.

The control volume method was initially evaluated by Walker et al. [40] to quantify flow through an orifice in vitro. A very good agreement was found between the measured and the true flow rates $(r=0.99)$. Subsequently, a detailed in vitro study [18] was performed to investigate for the effects of control volume size, valve geometry, and aortic outflow on the accuracy of the method in the quantification of the regurgitant flow. The results showed that a properly sized control volume whose boundary voxels excluded the region of flow acceleration and velocity aliasing too close to the orifice provided accurate measurements of the regurgitant flow (Fig. 9). A small control volume provided erroneous results, because the control volume boundaries were in the region of flow acceleration and velocity aliasing. A very large control volume generally provided inaccurate results due to reduced velocity sensitivity far from the orifice. Aortic outflow, orifice shape, and valve geometry did not affect the accuracy of the control volume measurements [18]. The effects of the motion of the mitral valve during the cardiac cycle

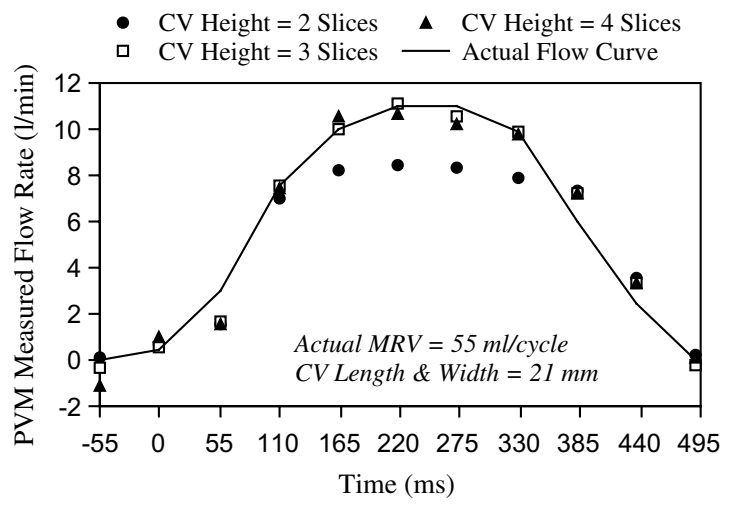

Fig. 9. Measured regurgitant flow waveform using different control volume $(\mathrm{CV})$ heights for a severe mitral regurgitation experimental simulation (mitral regurgitant volume $(\mathrm{MRV})=$ $55 \mathrm{ml} /$ beat). From the orientation of the slices in Fig. 8, the CV height is controlled by the number of slices included in the calculation. on the accuracy of the control volume measurements are significant [41]. Correction must be performed to account for the velocity of motion of the valve leaflets. The problem of cardiac motion may be eliminated with corrective techniques, such as the one described in the case of aortic regurgitation that involves an adaptation of slice position based on the motion of the basal cardiac plane [37]. This approach was implemented in a preliminary study in three patients with mitral regurgitation [38]. The mitral regurgitant volume was determined from the difference between the mitral diastolic inflow volume and the aortic systolic flow volume. The mitral annulus moved approximately $12 \mathrm{~mm}$ in the through-slice direction. The difference in the regurgitant volume between implementing and not implementing the corrective technique was approximately $19 \%$.

By investigating the effects of additional physiological factors (such as the motion of the leaflets, the presence of the chordae, etc.) and developing /implementing proper motion corrective procedures, the control volume method has the potential to be clinically established as a diagnostic approach in mitral regurgitation. With the development of ultra-fast MR PVM acquisition techniques, the time to acquire all three components of the velocity of blood in a number of slices will be short enough to allow a complete examination within a reasonable amount of time.

\section{Total cavo-pulmonary connection}

Congenital heart disease is the most prevalent type of heart disease among children, commonly resulting in single-ventricle physiology. Surgical repair is essentially the only survival option. The initial procedure was the Fontan operation performed in 1971 [42], which involved a connection of the superior vena cave (SVC) to the distal end of the right pulmonary artery (RPA), and a connection of the right atrial appendage to the proximal end of the RPA. Since 1971, the atriopulmonary Fontan procedure has been modified to improve the flow energetics of the new circulation. Currently, the most promising (and preferred) modification of the initial Fontan oper- 


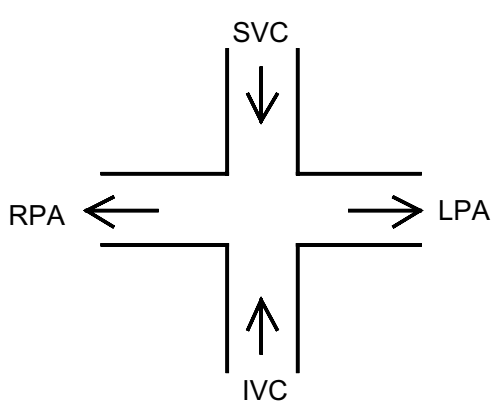

Fig. 10. A simplistic schematic of the total cavo-pulmonary connection. The superior vena cava (SVC) and the inferior vena cava (IVC) are surgically connected to the right pulmonary artery (RPA) and the left pulmonary artery (LPA). Blood flows from the SVC and IVC to the lungs via RPA and LPA.

ation is the total cavo-pulmonary connection (TCPC) (Fig. 10), which involves a (multi-stage) surgical connection of the SVC and the inferior vena cava (IVC) directly to the RPA [43]. The procedure shows evidence of improved performance over the atrio-pulmonary connections.

The superiority of TCPC over the atrio-pulmonary connection is partially due to the more streamlined flow patterns in TCPC, with less flow disturbances and, thus, lower fluid energy losses [43-46]. Optimization of the TCPC has been investigated through several in vitro, numerical, and in vivo studies, focusing on the reduction of the energy losses of blood flow through the connection and providing proper blood flow distribution to the lungs. The optimal design should prevent strong flow collisions, allowing at the same time adequate IVC flow to both the RPA and the LPA, as shown by in vitro and numerical studies [47-49]. One way to avoid a direct caval flow collision (and conserve energy) is to introduce an offset between the points of SVC and IVC connection with the RPA, as shown in vitro and in vivo [5053]. Addition of flaring at the connection site further improves the energetics of the system reducing the energy losses as much as $68 \%$ due to smoother transition of SVC and IVC flows to the pulmonary arteries [48,51-53].

To evaluate the energetics of the TCPC, the mechanical energy losses can be calculated via a control volume analysis (according to which the "energy loss" is equal to "mechanical energy IN" minus "mechanical energy OUT"). The practical limitation of this approach is that it requires invasive pressure measurements (to determine the potential part of the mechanical energy). This requirement makes the control volume approach less appealing clinically. A non-invasive technique to provide the energy loss in the TCPC is preferable.

MR techniques including MR PVM have been used to study Fontan cases [52-58] offering significant information that may assist in post-operative patient monitoring. Clinical TCPC studies have provided quantitative information for the distribution of blood from the SVC and IVC to the LPA and RPA [54,56]. The advantage of MR PVM being able to measure multi-directional blood velocity components $[52,53,57,58]$ has provided the ability to generate dynamic velocity vector maps in vitro [52] and in patients [53,57,58], showing (qualitatively) the fluid dynamic superiority (more unidirectional and less variable flow) of the TCPC over the atrio-pulmonary connection [57] and the importance of the presence of caval offset and flaring at the connection [53,54]. The accuracy of the information in the MR velocity vector plots is high as shown in vitro by comparing the MR PVM vector plots (Fig. 11) with those

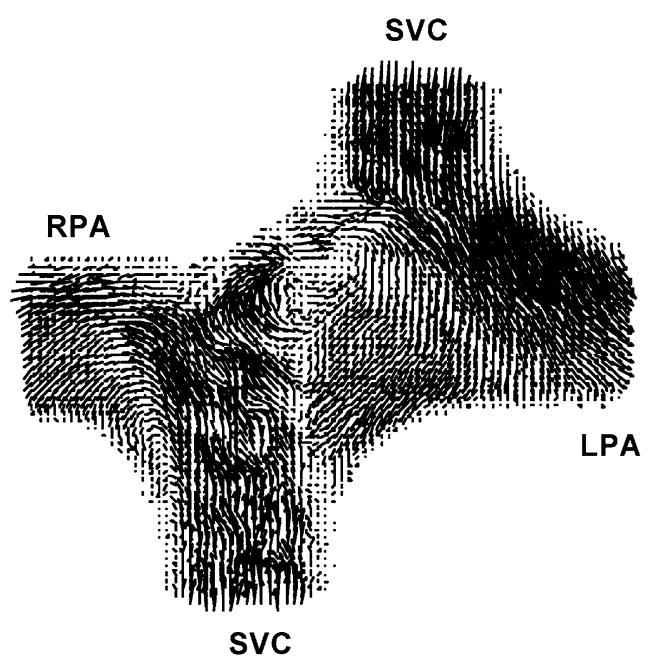

Fig. 11. A velocity vector plot in a model of the total cavopulmonary connection, constructed by combining two of the three measured spatial velocity components with MR PVM. 
from digital particle image velocimetry and with traditional particle flow visualization data [52]. There was agreement in the main features of the flow field (stagnation points, flow separation points, regions with secondary flows, etc.). A preliminary in vivo study in twelve TCPC patients using three-directional MR PVM provided blood velocity vector plots with clear visualization of fluid mechanic features (streamlined flow, flow swirling) [53]. The in vivo findings were in general agreement with previously described in vitro results $[51,52]$.

In addition to the qualitative flow field information that MR PVM provides, the three-directional velocity data may be used to calculate the viscous dissipation, which, according to fluid mechanics theory, is equal to the mechanical energy loss in a flow field such as that in the TCPC [59]. The combination of the semi-qualitative information from the velocity vector-plots and the quantitative information on the energy loss can provide better monitoring of the success of a specific TCPC design post-operatively as well as guide cardiologists and cardiac surgeons to make proper treatment decisions.

\section{Rapid magnetic resonance phase velocity mapping}

Conventional unsegmented $k$-space MR PVM is performed using the gradient-echo sequence shown in Fig. 1, with a bipolar velocity-encoding gradient in the desired direction for velocity measurements. Because of the arterial flow pulsatility, multiple measurements are acquired during the cardiac cycle. The MR scanner can be triggered by the electrocardiogram (ECG) signal of the human subject to identify the beginning of the cardiac cycle. Then, a number of acquisitions are performed at different time points (time phases) throughout the cycle. Only one line of $k$-space for each time phase is acquired per heart beat. As a result, the acquisition of a single velocity measurement typically requires a few minutes (depending on heart rate and imaging parameters). Since blood flow quantification is usually only part of a complete cardiac MR examination and sincenew clinical protocols to diagnose valvular disease and the energetics of TCPC involve multiple velocity acquisitions $[18,41]$, the unsegmented $k$-space technique becomes less practical clinically.

With the development of rapid imaging sequences, a segment of multiple $k$-space lines (instead of a single line) can be acquired very rapidly per time phase. Consequently, the acquisition can be performed in seconds instead of minutes, with high temporal and spatial resolution [60-64]. If velocity-encoding gradients are added to the regular sequence, the flow velocity can be measured rapidly. Systematic in vitro $[65,66]$ and in vivo [67] studies have shown that segmented $k$-space MR PVM is very accurate in quantifying flow from through-plane velocity measurements, under a variety imaging parameters. The in vitro study involved flow quantification from throughplane velocity data in straight tubes, under a variety of steady and pulsatile flow conditions. The effect of the number of $k$-space lines per segment was studied by examining the unsegmented sequence and three segmented $k$-space schemes, with five [65], seven [66] and nine [65] $k$-space lines per segment. Results showed that both segmented sequences provided very accurate flow quantification (errors $<5 \%$ ) under both steady and pulsatile flow conditions ( $p$-value $\gg 0.05$ ). Flow measurements in the human ascending aorta using the unsegmented sequence and the segmented sequence with seven and nine lines per segment [67] showed very close agreement ( $p$-value $\gg 0.05$ ) between the two segmented and the unsegmented technique. These results suggest that ultra-fast MR PVM has great potential for blood flow quantification clinically.

\section{Conclusion}

This review focused on magnetic resonance phase velocity mapping as a velocimetric and flowmetric technique. Specific clinical problems, such as the diagnosis of heart valve regurgitation and the evaluation of the energetic performance of the total cavo-pulmonary connection, for which MR PVM has great potential as a diagnostic and monitoring tool, were discussed. The potential for ultra-fast acquisitions was finally addressed. 
As the clinical interest in more detailed quantitative information about blood flow increases, the need for techniques such MR PVM becomes more apparent. This need combined with the continuous progress in MR technology promises even broader applications of MR PVM not only in the clinical but also in non-biomedical fields.

\section{References}

[1] P.R. Moran, A flow velocity zeugmatographic interlace for NMR imaging in humans, Magnetic Resonance Imaging 1 (1982) 197-203.

[2] S.R. Underwood, D.N. Firmin, R.H. Klipstein, R.S.O. Rees, D.B. Longmore, Magnetic resonance velocity mapping: Clinical application of a new technique, British Heart Journal 57 (1987) 404-412.

[3] R.H. Klipstein, D.N. Firmin, S.R. Underwood, R.S.O. Rees, D.B. Longmore, Blood flow patterns in the human aorta studied by magnetic resonance, British Heart Journal 58 (1987) 316-323.

[4] R.H. Mohiaddin, P.D. Gatehouse, D.N. Firmin, Exerciserelated changes in aortic flow measured with spiral echoplanar MR velocity mapping, Journal of Magnetic Resonance Imaging 5 (1995) 159-163.

[5] C. Kondo, G.R. Caputo, R. Semelka, E. Foster, A. Shimakawa, C.B. Higgins, Right and left ventricular stroke volume measurements with velocity-encoded cine MR imaging: In-vitro and in-vivo validation, American Journal of Roentgenology 157 (1991) 9-16.

[6] L.R. Pelc, N.J. Pelc, S.C. Rayhill, L.J. Castro, G.H. Glover, R.J. Herfkens, D.C. Miller, R.B. Jeffrey, Arterial and venous blood flow: Noninvasive quantitation with MR imaging, Radiology 185 (1992) 809-812.

[7] H.G. Bogren, M.H. Buonocore, Blood flow measurements in the aorta and major arteries with MR velocity mapping, Journal of Magnetic Resonance Imaging 4 (1994) 119-130.

[8] G.P. Chatzimavroudis, J.N. Oshinski, R.H. Franch, R.I. Pettigrew, P.G. Walker, A.P. Yoganathan, Quantification of the aortic regurgitant volume with magnetic resonance phase velocity mapping: A clinical investigation of the importance of imaging slice location, Journal of Heart Valve Disease 7 (1998) 94-101.

[9] P.J. Kilner, G.Z. Yang, R.H. Mohiaddin, D.N. Firmin, D.B. Longmore, Helical and retrograde secondary flow patterns in the aortic arch studied by three-directional magnetic resonance velocity mapping, Circulation 88 (1993) 2235-2247.

[10] A.M. Corbett, R.J. Phillips, R.J. Kauten, K.L. McCarthy, Magnetic resonance imaging of concentration and velocity profiles of pure liquids and solid suspensions in rotating geometries, Journal of Rheology 39 (1995) 907-924.

[11] P. Mansfield, R. Bowtell, S. Blackband, D.N. Guilfoyle, Magnetic resonance imaging: Applications of novel meth- ods in studies of porous media, Magnetic Resonance Imaging 10 (1992) 741-746.

[12] M.D. Mantle, A.J. Sederman, L.F. Gladden, Single- and two-phase flow in fixed-bed reactors: MRI flow visualisation and lattice-Boltzmann simulations, Chemical Engineering Science 56 (2001) 523-529.

[13] M.M. Britton, P.T. Callaghan, NMR velocimetry study of the temperature dependent rheology of butter, semisoft butter and margarine, Journal of Texture Studies 31 (2000) 245-255.

[14] D.F. Arola, G.A. Barrall, R.L. Powell, K.L. McCarthy, M.J. McCarthy, Use of nuclear magnetic resonance imaging as a viscometer for process monitoring, Chemical Engineering Science 52 (1997) 2049-2057.

[15] J.L. Duerk, P.M. Pattany, In-plane flow velocity quantification along the phase encoding axis in MRI, Magnetic Resonance Imaging 6 (1988) 321-333.

[16] D. Meier, S. Maier, P. Bosiger, Quantitative flow measurements on phantoms and on blood vessels with MR, Magnetic Resonance in Medicine 8 (1988) 25-34.

[17] G.P. Chatzimavroudis, P.G. Walker, J.N. Oshinski, R.H. Franch, R.I. Pettigrew, A.P. Yoganathan, Slice location dependence of aortic regurgitation measurements with MR phase velocity mapping, Magnetic Resonance in Medicine 37 (1997) 545-551.

[18] G.P. Chatzimavroudis, J.N. Oshinski, R.I. Pettigrew, P.G. Walker, R.H. Franch, A.P. Yoganathan, Quantification of mitral regurgitation with magnetic resonance phase velocity mapping using a control volume method, Journal of Magnetic Resonance Imaging 8 (1998) 577-582.

[19] K.W. Moser, E.C. Kutter, J.G. Georgiadis, R.O. Buckius, H.D. Morris, J.R. Torczynski, Velocity measurements of flow through a step stenosis using magnetic resonance imaging, Experiments in Fluids 29 (2000) 438447.

[20] G.P. Chatzimavroudis, J.N. Oshinski, R.H. Franch, P.G. Walker, A.P. Yoganathan, R.I. Pettigrew, Evaluation of the precision of magnetic resonance phase velocity mapping for blood flow measurements, Journal of Cardiovascular Magnetic Resonance 3 (2001) 11-19.

[21] M.-C. Dulce, G.H. Mostbeck, M. O'Sullivan, M. Cheitlin, G.R. Caputo, C.B. Higgins, Severity of aortic regurgitation: Interstudy reproducibility of measurements with velocity-encoded cine MR imaging, Radiology 185 (1992) 235-240.

[22] C.H. Croft, K. Lipscomb, K. Mathis, B.G. Firth, P. Nicod, G. Tilton, M.D. Winnford, L.D. Hillis, Limitations of qualitative angiographic grading in aortic or mitral regurgitation, The American Journal of Cardiology 53 (1984) 1593-1598.

[23] S. Holm, P. Eriksson, K. Karp, G. Osterman, D. Teien, Quantitative assessment of aortic regurgitation by combined two-dimensional, continuous-wave and colour flow Doppler measurements, Journal of Internal Medicine 231 (1992) 115-121.

[24] G.J. Perry, F. Helmcke, N.C. Nanda, C. Byard, B. Soto, Evaluation of aortic insufficiency by Doppler color flow 
mapping, Journal of the American College of Cardiology 9 (1987) 952-959.

[25] S. Wagner, W. Auffermann, P. Buser, T.H. Lim, B. Kircher, P. Pflugfelder, C.B. Higgins, Diagnostic accuracy and estimation of the severity of valvular regurgitation from the signal void on cine magnetic resonance images, American Heart Journal 118 (1989) 760-767.

[26] J.-I. Suzuki, G.R. Caputo, C. Kondo, C.B. Higgins, Cine MR imaging of valvular heart disease: Display and imaging parameters affect the size of the signal void caused by valvular regurgitation, American Journal of Roentgenology 155 (1990) 723-727.

[27] A.L. Taylor, E.J. Eichhorn, M.E. Brickner, R.C. Eberhart, P.A. Grayburn, Aortic valve morphology: An important in vitro determinant of proximal regurgitant jet width by Doppler color flow mapping, Journal of the American College of Cardiology 16 (1990) 405-412.

[28] T. Reynolds, J. Abate, A. Tenney, M.G. Warner, The JH/ LVOH method in the quantification of aortic regurgitation: How the cardiac sonographer may avoid an important potential pitfall, Journal of the American Society of Echocardiography 4 (1991) 105-108.

[29] S. Globits, H. Mayr, A. Neuhold, D. Globits, H. Frank, Quantitative assessment of aortic regurgitation by magnetic resonance imaging, European Heart Journal 13 (1992) 78-83.

[30] U. Sechtem, P.W. Pflugfelder, M.M. Cassidy, R.D. White, M.D. Cheitlin, N.B. Schiller, C.B. Higgins, Mitral or aortic regurgitation: Quantification of regurgitant volumes with cine MR imaging, Radiology 167 (1988) 425-430.

[31] F. Recusani, G.S. Bargiggia, A.P. Yoganathan, A. Raisaro, L.M. Valdes-Cruz, H.-W. Sung, C. Bertucci, M. Gallati, V.A. Moises, I.A. Simpson, L. Tronconi, D.J. Sahn, A new method for quantification of regurgitant flow rate using color Doppler flow imaging of the flow convergence region proximal to a discrete orifice: An invitro study, Circulation 83 (1991) 594-604.

[32] J. Hopmeyer, A.A. Fontaine, S. Yang, R.A. Levine, A.P. Yoganathan, The effect of aortic outflow on the quantification of mitral regurgitation by the flow convergence method, Journal of the American Society of Echocardiography 9 (1996) 44-57.

[33] G.B. Cranney, C.S. Lotan, G.M. Pohost, Evaluation of aortic regurgitation by nuclear magnetic resonance imaging, Current Problems in Cardiology 15 (1990) 87-114.

[34] L. Søndergaard, K. Lindvig, P. Hildebrandt, T. Joen, C. Thompsen, F. Stahlberg, O. Henriksen, Aortic regurgitation quantified by magnetic resonance velocity mapping, in: 11th Annual Meeting of SMRM, Berlin, Germany, vol. 1, 1992, p. 523.

[35] N. Honda, K. Machida, M. Hashimoto, T. Mamiya, T. Takahashi, T. Kamano, A. Kashimada, Y. Inoue, S. Tanaka, N. Yoshimoto, H. Matsuo, Aortic regurgitation: Quantitation with MR imaging velocity mapping, Radiology 186 (1993) 189-194.

[36] L. Søndergaard, K. Lindvig, P. Hildebrandt, C. Thompsen, F. Stahlberg, T. Joen, O. Henriksen, Quantification of aortic regurgitation by magnetic resonance velocity mapping, American Heart Journal 125 (1993) 1080-1090.

[37] S. Kozerke, M.B. Scheidegger, E.M. Pedersen, P. Boesiger, Heart motion adapted cine phase-contrast flow measurements through the aortic valve, Magnetic Resonance in Medicine 42 (1999) 970-978.

[38] S. Kozerke, J. Schwitter, E.M. Pedersen, P. Boesiger, Aortic and mitral regurgitation: Quantification using moving slice velocity mapping, Journal of Magnetic Resonance Imaging 14 (2001) 106-112.

[39] N. Fujita, A.F. Chazouilleres, J.J. Hartiala, M. O’Sullivan, P. Heidenreich, J.D. Kaplan, H. Sakuma, E. Foster, G.R. Caputo, C.B. Higgins, Quantification of mitral regurgitation by velocity-encoded cine nuclear magnetic resonance imaging, The American Journal of Cardiology 23 (1994) 951-958.

[40] P.G. Walker, S. Oyre, E.M. Pedersen, K. Houlind, F.S.A. Guenet, A.P. Yoganathan, A new control volume method for calculating valvular regurgitation, Circulation 92 (1995) 579-586.

[41] P.G. Walker, K. Houlind, C. Djurhuus, W.Y. Kim, E.M. Pedersen, Motion correction for the quantification of mitral regurgitation using the control volume method, Magnetic Resonance in Medicine 43 (2000) 726-733.

[42] F. Fontan, E. Baudet, Surgical repair of tricuspid atresia, Thorax 26 (1971) 240-248.

[43] M.R. de Leval, P. Kilner, M. Gewillig, C. Bull, Total cavopulmonary connection: A logical alternative to atriopulmonary connection for complex Fontan operations, Journal of Thoracic and Cardiovascular Surgery 96 (1988) 682-695.

[44] Y.H. Kim, P.G. Walker, A.A. Fontaine, S. Panchal, A.E. Ensley, J.N. Oshinski, S. Sharma, B. Ha, C.L. Lucas, A.P. Yoganathan, Hemodynamics of the Fontan connection: An in-vitro study, Journal of Biomechanical Engineering 117 (1995) 423-428.

[45] A.C. Lardo, P.J. del Nido, S.A. Webber, I. Friehs, E.G. Cape, Hemodynamic effect of progressive right atrial dilatation in atriopulmonary connections, Journal of Thoracic and Cardiovascular Surgery 114 (1997) 2-8.

[46] H.T. Low, Y.T. Chew, C.N. Lee, Flow studies on atriopulmonary and cavopulmonary connections of the Fontan operations for congenital heart defects, Journal of Biomedical Engineering 15 (1993) 303-307.

[47] A.C. Lardo, S.A. Webber, A. Iyengar, P.J. del Nido, I. Friehs, E.G. Cape, Bidirectional superior cavopulmonary anastomosis improves mechanical efficiency in dilated atriopulmonary connections, Journal of Thoracic and Cardiovascular Surgery 118 (1999) 681-691.

[48] G. Dubini, M.R. de Leval, R. Pietrabissa, F.M. Montevecchi, R. Fumero, A numerical fluid mechanical study of repaired congenital heart defects: Application to the total cavopulmonary connection, Journal of Biomechanics 29 (1996) 111-121.

[49] F. Migliavacca, M.R. de Leval, G. Dubini, R. Pietrabissa, R. Fumero, Computational fluid dynamic simulations of cavopulmonary connections with an extracardiac lateral 
conduit, Medical Engineering and Physics 21 (1999) 187193.

[50] S. Sharma, S. Goudy, P. Walker, S. Panchal, A. Ensley, K. Kanter, V. Tam, D. Fyfe, A. Yoganathan, In vitro flow experiments for determination of optimal geometry of total cavopulmonary connection for surgical repair of children with functional single ventricle, Journal of the American College of Cardiology 27 (1996) 1264 1269.

[51] A.E. Ensley, P. Lynch, G.P. Chatzimavroudis, C. Lucas, S. Sharma, A.P. Yoganathan, Toward designing the optimal total cavopulmonary connection: An in vitro study, Annals of Thoracic Surgery 68 (1999) 1384-1390.

[52] A.E. Ensley, A. Ramuzat, T.M. Healy, G.P. Chatzimavroudis, C. Lucas, S. Sharma, R. Pettigrew, A.P. Yoganathan, Fluid mechanic assessment of the total cavopulmonary connection using magnetic resonance phase velocity mapping and digital particle image velocimetry, Annals of Biomedical Engineering 28 (2000) 11721183.

[53] S. Sharma, A.E. Ensley, K. Hopkins, G.P. Chatzimavroudis, T.M. Healy, V.K.H. Tam, K.R. Kanter, A.P. Yoganathan, In vivo flow dynamics of the total cavopulmonary connection from three-dimensional multislice magnetic resonance imaging, Annals of Thoracic Surgery 71 (2001) 889-898.

[54] K. Houlind, E.V. Stenbog, K.E. Sorensen, K. Emmertsen, O.K. Hansen, L. Rybro, V.E. Hjortdal, Pulmonary and caval flow dynamics after total cavopulmonary connection, Heart 81 (1999) 67-72.

[55] S. Rebergen, J. Ottenkamp, J. Doornbos, E. van der Wall, J. Chin, A. de Roos, Postoperative pulmonary flow dynamics after Fontan surgery: Assessment with nuclear magnetic resonance velocity mapping, Journal of the American College of Cardiology 21 (1993) 123-131.

[56] M.A. Fogel, P.M. Weinberg, J. Rychik, A. Hubbard, M. Jacobs, T.L. Spray, J. Haselgrove, Caval contribution to flow in the branch pulmonary arteries of Fontan patients with a novel application of magnetic resonance presaturation pulse, Circulation 99 (1999) 1215-1221.

[57] E. Be'eri, S.E. Maier, M.J. Landzberg, T. Chung, T. Geva, In vivo evaluation of Fontan pathway flow dynamics by multidimensional phase-velocity magnetic resonance imaging, Circulation 98 (1998) 2873-2882.

[58] V.L. Morgan, T.P. Graham, R.J. Roselli, C.H. Lorenz, Alterations in pulmonary artery flow patterns and shear stress determined with three-dimensional phase contrast magnetic resonance imaging in Fontan patients, Journal of
Thoracic and Cardiovascular Surgery 116 (1998) 294 304.

[59] T.M. Healy, C. Lucas, A.P. Yoganathan, Noninvasive fluid dynamic power loss assessments for total cavopulmonary connections using the viscous dissipation function: A feasibility study, Journal of Biomechanical Engineering 123 (2001) 317-324.

[60] C. Thomsen, M. Cortsen, L. Søndergaard, O. Henriksen, F. Ståhlberg, A segmented $k$-space velocity mapping protocol for quantification of renal artery blood flow during breath-holding, Journal of Magnetic Resonance Imaging 5 (1995) 393-401.

[61] C.P. Davis, P.-F. Liu, M. Hauser, S.C. Göhde, G.K. von Schulthess, J.F. Debatin, Coronary flow and coronary flow reserve measurements in humans with breath-held magnetic resonance phase contrast velocity mapping, Magnetic Resonance in Medicine 37 (1997) 537-544.

[62] M. Bock, S.O. Schoenberg, L.R. Schad, M.V. Knopp, M. Essig, G. van Kaick, Interleaved gradient-echo planar (IGEPI) and phase contrast CINE-PC flow measurements in the renal artery, Journal of Magnetic Resonance Imaging 8 (1998) 889-895.

[63] V.-P. Poutanen, R. Kivisaari, A.-M. Häkkinen, S. Savolainen, P. Hekali, C.-G. Standertskjöld-Nordenstam, Multiphase segmented $k$-space velocity mapping in pulsatile flow waveforms, Magnetic Resonance Imaging 16 (1998) 261-269.

[64] E. Laffon, R. Lecesne, V. de Ledinghen, N. Valli, P. Couzigou, F. Laurent, J. Drouillard, D. Ducassou, J.-L. Barat, Segmented 5 versus nonsegmented flow quantitation: Comparison of portal vein flow measurements, Investigative Radiology 34 (1999) 176-180.

[65] H. Zhang, S.S. Halliburton, J.R. Moore, O.P. Simonetti, P.R. Schvartzman, R.D. White, G.P. Chatzimavroudis, Ultra-fast flow quantification with segmented $k$-space magnetic resonance phase velocity mapping, Annals of Biomedical Engineering 30 (2002) 120-128.

[66] H. Zhang, S.S. Halliburton, J.R. Moore, O.P. Simonetti, P.R. Schvartzman, R.D. White, G.P. Chatzimavroudis, Accurate quantification of steady and pulsatile flow with segmented $k$-space magnetic resonance velocimetry, Experiments in Fluids 33 (2002) 458-463.

[67] G.P. Chatzimavroudis, H. Zhang, S.S. Halliburton, J.R. Moore, O.P. Simonetti, P.R. Schvartzman, A.E. Stillman, R.D. White, Clinical blood flow quantification with segmented $k$-space magnetic resonance phase velocity mapping, Journal of Magnetic Resonance Imaging 17 (2003) 65-71. 